\title{
Overview of Telemedicine and Sleep Disorders
}

Jaspal Singh, MD, MHA, MHS ${ }^{a, *}$, Nikky Keer, DO ${ }^{b}$

\section{KEYWORDS}

$\bullet$ Telemedicine $\bullet$ Telehealth $\bullet$ Virtual care $\bullet$ Sleep $\bullet$ Download $\bullet$ Data integration

\section{KEY POINTS}

- There is a clear need for the sleep medicine provider to consider telehealth use, and find means for incorporating, integrating, and expanding telehealth strategies.

- The technology behind telemedicine integration is often less of a consideration than strategic design of the telemedicine practice (workflow design, logistics, and communication).

- The telehealth industry's rapid and expansive growth, coupled with evolving legislation and reimbursement schemes, requires attention and consideration when defining the technology and applications.

- Individual articles provide a more comprehensive examination of the various aspects of sleep telehealth adoption and integration.

- Important questions related to telehealth still need to be answered, including what is the true impact of telehealth on quality, access, and efficiencies of sleep medicine? What is the impact of telehealth on individual patient and provider experiences?

\section{INTRODUCTION}

We are delighted to introduce this edition of Sleep Medicine Clinics, which focuses on telemedicine and its applications, whereby multiple authors provide important and unique insights to the issues surrounding sleep telehealth as this field evolves. In this introduction, we provide a clinical, organizational, and philosophic context to the incorporation of sleep telehealth. To us and many others, telemedicine for sleep disorders is a natural fit, because:

- There is a large unmet patient need for sleep specialists that may be partially allayed by the expansion of telemedicine.

- Diagnostic and treatment algorithms of several common disorders like obstructive sleep apnea are often data driven, enabling sound clinical decision making, potentially even in the absence of a physical examination.

- Sleep clinicians generally rely less on discrete physical examination findings than many other specialists.

- Telemedicine may minimize some of the traditional barriers that have hindered the growth of sleep medicine as a specialty.

Given these and other issues related to rapid technological adoption by society in general, sleep medicine seems poised to build on this platform of patient care delivery.

\section{CONTENT}

The telemedicine industry is growing, and even before the coronavirus disease-2019 (COVID-19)

\footnotetext{
a Atrium Health and Levine Cancer Institute, Carolinas Medical Center and Atrium Health, 1000 Blythe Boulevard, 506B Medical Education Building, Charlotte, NC 28203, USA; b Division of Pulmonary, Critical Care, and Sleep Medicine, Department of Medicine, University of Tennessee HSC COM at Memphis, Suite H316B, 956 Court Avenue, Memphis, TN 38163, USA

* Corresponding author.

E-mail address: Jaspal.Singh@atriumhealth.org

Twitter: @Singh011Jaspal (J.S.); @nikkykeer (N.K.)
} 
pandemic, the market was expected to hit $\$ 53.1$ billion by 2026 in a recent report from Acumen Research and Consulting. ${ }^{1}$ Fueled by faster data streaming options, better camera resolution, and enhanced software features, the technical aspects seem positioned optimally for telemedicine to succeed. In fact, since COVID-19 and the widespread adoption of telemedicine to provide care while practicing social distancing, we expect those figures to skyrocket. ${ }^{2}$ The frenetic pace at which primary care and specialties adopt telemedicine practices has been nothing short of breathtaking for telemedicine enthusiasts. It is also quite possible that the COVID-19 pandemic and the Medicare CARES Act have changed the face of telemedicine for the foreseeable future.

So, if adequate technology is ubiquitous, why has telemedicine for sleep practices been so slow to evolve? It has been slowed down not only by previous issues of low reimbursement and slow adoption of technology, but also several key issues related to workflow disruption, behavioral and psychological issues, and of course disparities in access to technology. ${ }^{3}$ As such, the array of services, user functionalities, and payment and workflow schemes have left many confused, and perhaps overwhelmed, by where to begin. There are many unique factors to health care regulation, many players and stakeholders, and a host of issues related to change management difficulties. Thus, it has been difficult to identify clear paths to success as the pace of the technology, consumer demand, and regulatory and financial issues are continually evolving. Such rapid and constant change are almost akin to playing a high-stakes game while one is actively learning the rules; moreover, the rules keep changing. With all these factors, it is no wonder many sleep medicine clinicians have been less keen to adopt and incorporate telehealth.

Sleep medicine has been exposed previously to large-scale disruption, because home sleep apnea testing was met by years of intense resistance. ${ }^{4,5}$ This change finally led to broader acceptance and integration into the daily practice of a sleep practitioner, benefitting a countless number of patients. Many would suggest sleep medicine as a field benefitted from incorporation of new technology. In this vein, we would in fact argue that it is once again in the best interest of the field to accept this tidal wave of change by broad adoption and incorporation of telehealth into the sleep provider's clinical operations. We believe this has the potential to clearly benefit patients, sleep practices, and create much larger and broader impact through several potential mechanisms as discussed in this issue of Sleep Medicine Clinics (Fig. 1). However, the logistics of telemedicine, including its nuances, need to be understood. Importantly, one must also consider a patientcentered approach while maintaining fiscal, ethical, and legal responsibilities.

\section{Benefits of Sleep \\ Telehealth from \\ Patient Perspective}

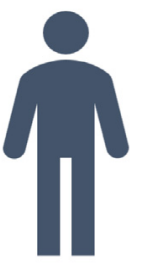

- Convenient visits

- Fewer barriers to care

- Use existing tools to monitor

- Personalize care through technology

- Timely feedback and interaction
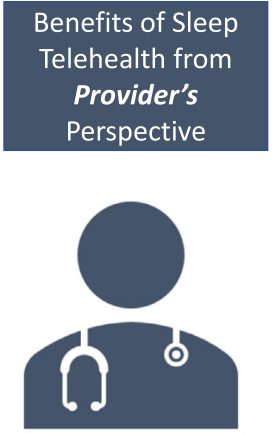

- Minimize distance travel

- Customize apps to practice needs

- Potential to manage larger panel

- Increased abilities to supervise APPs, incorporate team members

- Unique opportunities for coding, billing, practice growth

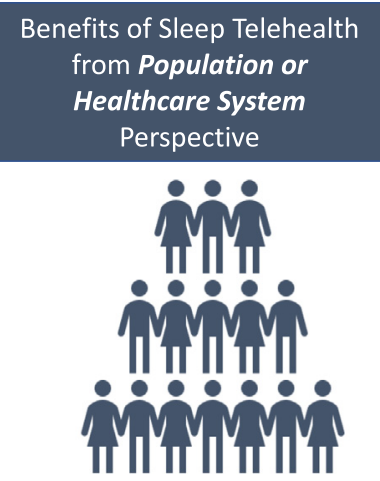

- Provide effective team-based communication and coordination

- Integrate care with EHR, data analytics

- Incorporate decision-support systems and $\mathrm{Al}$

- Drive large-scale initiative and complex care mechanisms

Fig. 1. The potential benefits of sleep telehealth from different perspectives. The use of telehealth has the ability to benefit a number of different key participants in unique ways. Al, artificial intelligence; APP, advanced practice provider (eg, nurse practitioner, physician assistant); EHR, electronic health record. 
As background, in 2015, the American Academy of Sleep Medicine published its Position Paper for the Use of Telemedicine for the Diagnosis and Treatment of Sleep Disorders, ${ }^{6}$ to seek more efficient and accessible ways to provide services beyond the traditional office model. The Position Paper noted that, at time of publication, the expansion of sleep telemedicine into all aspects of sleep disorder management was limited by technology resources and reimbursement and financial considerations, as well as a willingness of physicians, patients, and health care organizations to accept telemedicine as an alternative to in-office care. ${ }^{6}$ In this edition of Sleep Medicine Clinics, the editors have assembled a thoughtful group of experts who have a great deal of pragmatic experience in each of their areas, so that readers can learn other success factors (and some difficult lessons) in sleep telehealth use.

\section{Defining Sleep Telehealth}

Because telemedicine is often referred to mean different models of care delivery, a quick recap of the definitions might be worth noting. The American Telemedicine Association cites 4 key models of telemedicine services: synchronous and asynchronous (store-and-forward) telemedicine patient encounters, remote patient monitoring (RPM), and mobile health (mHealth) smartphone applications. Each of these receives a thorough examination in this edition, with pragmatic advice provided by each of the authors. For some basic definitions:

- Synchronous telemedicine refers to the delivery of a live, interactive encounter in which patients and providers are separated by distance but interact in real-time using videoconferencing as the core technology. Participants are separated by distance but interact synchronously with the provider performing interviews of the patient, and diagnostic and treatment options are addressed through live video interaction between the patient and the provider.

- Asynchronous (store-and-forward) telemedicine is defined as a non-real-time, technology-assisted exchange of structured information between a patient and provider with the intent to diagnose, treat, and/or triage. For example, a sleep medicine history with certain diagnostic/therapeutic data are collected at the point of care and transmitted to the sleep medicine provider for review. In turn, the sleep medicine specialist provides clinical advice via a written or electronic report to the referring provider within a reasonable time frame to make clinical decisions.
- $R P M$ refers to the use of digital devices to remotely collect physiologic data for interpretation and management of a patient under a specific treatment plan. For example, telemonitoring is routinely performed of positive airway pressure use; however, increasing RPM of physical activity, oximetry, or ambulatory blood pressure by the sleep clinician may also be considered. As such, this situation has to the potential to lead to personalized care, more rapid and frequent assessments, and perhaps even behavioral change. In addition, this technology may be used to do drive complex disease management to care for highrisk patients with a mix of cardiopulmonary and neuropsychiatric disorders. Such applications have been shown to be successful in a broad range of disease states.

- Mobile health applications, or mHealth, encompasses personal computer and smartphone applications that may be used to provide individuals with the behavioral and cognitive skills to manage a disease process. The application of sensors, mobile apps, and location tracking technology may not only enable simple behavioral changes, but also allow monitoring and intervention whenever and wherever acute and chronic medical conditions occur.

Arguably, RPM and mHealth models represent a heterogenous spectrum between asynchronous telemedicine and self-directed care mechanisms. However, these are worth mentioning now.

Importantly, the use of telehealth provides the opportunity therefore for responsive feedback from the patient and/or population, while setting the stage for more complex disease and population management schemes. It also creates interesting dilemmas as the lines become increasingly blurred between defining when a patient encounter begins and ends, which is why it is imperative to understand the technical, legal, regulatory, and financial environments in which one develops telehealth strategies.

\section{The Workforce Issue}

Incorporation of sleep telehealth has the inherent potential to allow the limited number of specialists in the workforce to serve the broader population. The Centers for Disease Control and Prevention estimate that 50 to 70 million of the general adult population in the United States suffers from chronic disease related to sleep deficiency and sleep disorders. The repercussions of sleep deficiency include disease burden, lost productivity and accidents, and an array of social determinants 
underlying health and health disparities. Fortunately, the general public is more aware than ever before of the importance of healthy sleep. Employers are placing a greater value on sleep and insurers, regulators, and legislators recognize the importance of diagnosing and treating sleep disorders. However, as of 2018, there are 6035 sleep medicine physicians board certified by the American Board of Medical Specialties. ${ }^{7}$ The reality is that this workforce is insufficient to meet the demands of the enormous population of patients who have a sleep disorder. By leveraging telemedicine capabilities, sleep physicians can access patients that who be in more rural or remote locations and increase access to subspecialties within sleep (eg, pediatric sleep experts, dental sleep experts), while allowing those practitioners to save time and costs associated with travel or distant clinics.

Importantly, telemedicine truly allows sleep providers to serve more as team leaders in the care of patients, allowing for potentially a greater population served. Studies conducted within closed health care systems such as the Veterans Administration system ${ }^{8}$ or Kaiser Permanente ${ }^{9}$ have provided data on the value of telehealth components for evaluation and treatment, and how these components are delivered. Telehealth use has led to dramatically reduced wait-times from referral to diagnosis and treatment of sleep-related breathing disorders as well as increased adherence to treatment, despite an increase in the volume of sleep consults and sleep studies performed..$^{8,9}$

Questions remain, however, about whether the workforce can adapt to these pressures. Will telemedicine allow these providers to adopt, sustain, and grow larger panels of patients? Will such providers be skilled and enabled to lead care teams through virtual access tools? Will regulations and payment schemes limit successes in different markets of this approach? These are important questions that need to be addressed from the workforce perspective on the use of telemedicine.

\section{Financial Considerations}

Reimbursement has been and is one of the most significant barriers to the implementation of telehealth nationwide. Encouragingly, state and federal legislation and regulation have been increasingly broadening access to telehealth services in recent years. In 2019, 48 state Medicaid programs were reimbursing for synchronous video-based telehealth. Additionally, 40 states and the District of Columbia had adopted substantive policies to expand telehealth coverage and reimbursement. Furthermore, the Centers for Medicare and Medicaid Services has shown a dedication to support these policies by continually expanding its fee schedule to reimburse newer forms of telehealth. For example, Current Procedural Terminology codes to support RPM of physiologic values were added to the 2019 Medicare Physician Fee Schedule. Even before the COVID19 pandemic, there had been rapid adoption, acceptance, and even demand of telehealth by both physicians and patients. A study of more than 29 billion private health care claim records found that national use of telehealth grew tremendously between 2014 and $2018 .^{10}$ In particular, the number of non-hospital-based provider-to-patient telehealth claims increased at a rate of an astonishing $1393 \%$, much greater than other types of telehealth claims (Fig. 2).

Investment opportunities in telehealth have also been rapidly expanding. Remarkably, digital health received nearly 1 in 10 venture dollars invested in the United States in 2019, totaling more than $\$ 7$ billion. ${ }^{11}$ Additionally, digital health companies are proving outcomes and cost validation, increasing the likelihood of success when these ventures enter the public market. As of January 1,2020 , the combined market cap of the 9 digital health companies that entered the public market in 2019 is a staggering $\$ 17$ billion. ${ }^{11}$ There are countless applications currently on the market to provide an array of telehealth solutions, and likely more to come soon. Importantly, in 2016 the American Academy of Sleep Medicine even launched its own sleep telemedicine platform, SleepTM. This platform provides services ranging from synchronous telemedicine encounters for sleep disordered breathing, cognitive behavioral therapy for insomnia as well as data management and coordination of care services for durable medical equipment. With the dizzying pace of technological innovation, the US Food and Drug Administration has enacted a Digital Health Innovation Action Plan to provide regulatory framework for medical device software, mHealth, and other platforms. The goal is to provide timely patient access to high-quality, safe, and effective medical technology.

Regardless of telehealth strategy and application(s), the financial implications for payers, industry, investors, and the consumers will be scrutinized closely. As much as telemedicine offers a lot to be gained, concerns of costs, integration, and usability still will remain. Moreover, the current reimbursement scheme by the Centers for Medicare and Medicaid Services is in response to the COVID-19 pandemic, which if the reimbursement changes afterward, or the health care sector economy collapses, ${ }^{12}$ then telemedicine may not be as easy to sustain in the current 
$0.1600 \%$

$0.1400 \%$

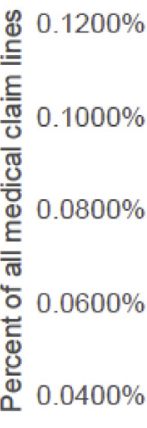

$0.0200 \%$

$0.0000 \%$
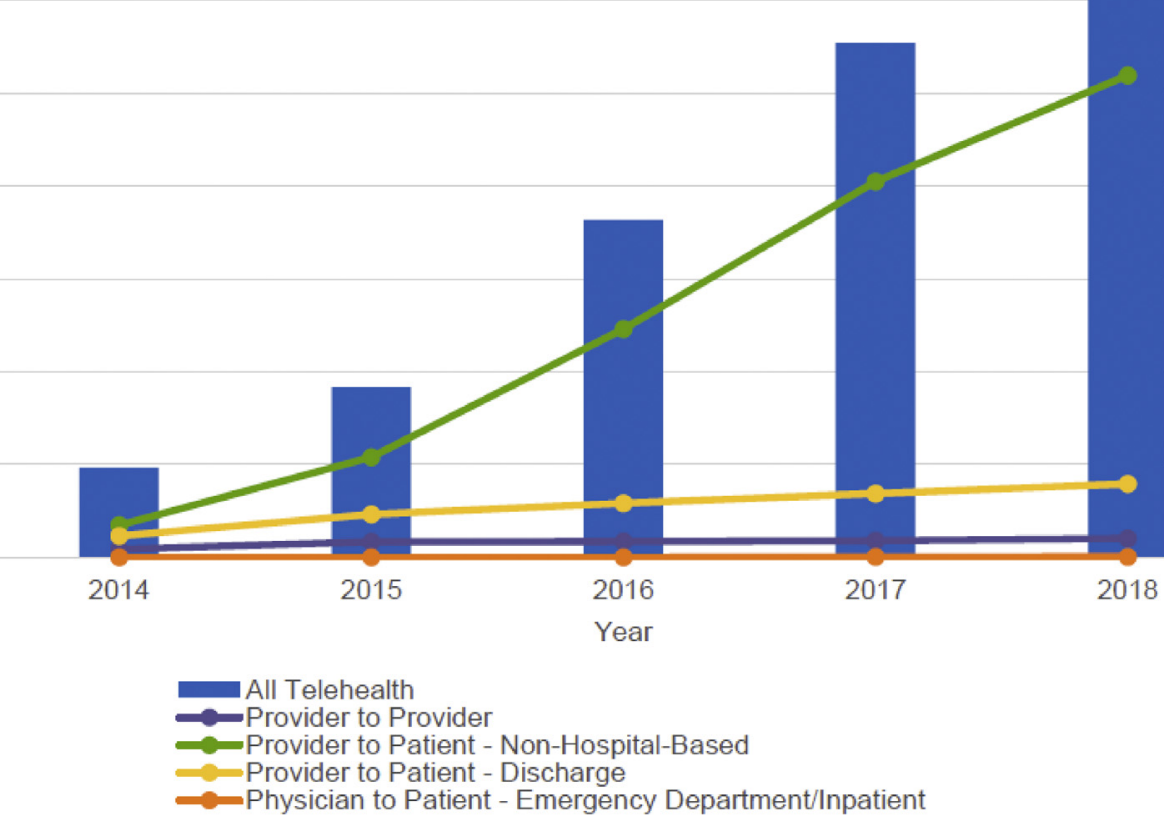

Fig. 2. Claim lines with telehealth usage by type as a percentage of all medical claim lines. (Source: "A Multilayered Analysis of Telehealth" [June 2019]. (C) FAIR Health, Inc. Reprinted with permission.)

projections. Much is evolving in this landscape as the financial sectors brace for a post-COVID-19 world, and sleep telemedicine is not immune to the market forces.

\section{How to Navigate This Issue of Sleep Medicine Clinics}

This issue of Sleep Medicine Clinics offers a complete review of key principles related to sleep telemedicine. After the introduction, this edition begins with implementation of synchronous telemedicine, a staple for most sleep telemedicine provider's approach. Therefore, understanding the practical aspects of the telemedicine or virtual visit is an imperative for the sleep professional. This is then followed by a thoughtful discussion of how one approaches the electronic health record, integration with data systems, and population management tools in sleep medicine. Subsequent articles expand on the introduction of consumer sleep technologies and device wearables mentioned elsewhere in this issue, providing the reader with insight into the rapid advancements that many sleep clinicians see daily in their offices. After this overview, further detail is provided regarding how to manage certain populations with common sleep disorders such as obstructive sleep apnea and insomnia, with the respective authors providing tremendous insight and pragmatic information including managing those with complex interrelated disease states. This issue also addresses how to use telehealth to better integrate nonphysician providers, facilitate team-based care, and even what unique teaching and research opportunities are afforded using telemedicine. Importantly, in-depth evaluations of regulatory, legal, and ethics issues as well as important principles related to coding, reimbursement, and financial considerations are highlighted. Last, the integration of predictive analytics as the concluding article leads us into the many possibilities that telemedicine with all its uses and applications may allow.

We are excited about this edition, particularly as each author is not only an accomplished leader, but also a skilled clinician. As such, each author has tried (and failed) at certain aspects of telemedicine care delivery, but each wants the next person to be successful in advancing the field as well as the care of any patient with a sleep disorder. 


\section{SUMMARY}

Telemedicine is growing rapidly in all aspects of medicine, and sleep medicine seems well-poised to adapt to this transformation of health care delivery. That said, there are numerous issues related to understanding the technologies, the interface, the workflow and the patient and provider experience. Last, providing value, and remaining fiscally, legally, and ethically responsible are important considerations with the sleep telemedicine. Therefore, in this issue of Sleep Medicine Clinics, a collection of articles has been assembled that address where our field currently is with respect to telemedicine and telehealth principles for many sleep disorders. It is our opinion that sleep telehealth will be synonymous and integrated with bedside care of the patient with sleep disorders, and that the time is now to incorporate telemedicine principles into one's sleep medicine practice. But there remain questions as to how and to what degree sleep telemedicine will affect the quality, access, and efficiencies of the care of the sleep patient, and to what lessons the sleep medicine field will learn and share along this journey.

\section{DISCLOSURE}

J. Singh is on the Physician Advisory Board of Somnoware Sleep Solutions, Inc.

\section{REFERENCES}

1. Smith R. Telehealth market to hit $\$ 53.1$ billion by 2026. Insurance Business America Web site. 2020. Available at: https://www.insurancebusinessmag. com/us/news/breaking-news/telehealth-market-tohit-53-1-billion-by-2026-report-213866.aspx. Accessed May 5, 2020.

2. Hollander JE, Carr BG. Virtually perfect? telemedicine for COVID-19. N Engl J Med 2020;382(18): 1679-81.
3. Kane CK, Gillis K. The use of telemedicine by physicians: still the exception rather than the rule. Health Aff (Millwood) 2018;37(12):1923-30.

4. Tan HL, Kheirandish-Gozal L, Gozal D. Pediatric home sleep apnea testing: slowly getting there! Chest 2015;148(6):1382-95.

5. Collop NA. Portable monitoring for the diagnosis of obstructive sleep apnea. Curr Opin Pulm Med 2008; 14(6):525-9.

6. Singh J, Badr MS, Diebert W, et al. American Academy of Sleep Medicine (AASM) position paper for the use of telemedicine for the diagnosis and treatment of sleep disorders. J Clin Sleep Med 2015; 11(10):1187-98.

7. Kiley JP, Twery MJ, Gibbons GH. The national center on sleep disorders research-progress and promise. Sleep 2019;42(6):zsz105.

8. Sarmiento KF, Folmer RL, Stepnowsky CJ, et al. National expansion of sleep telemedicine for veterans: the telesleep program. J Clin Sleep Med 2019;15(9): 1355-64.

9. Hwang D, Chang JW, Benjafield AV, et al. Effect of telemedicine education and telemonitoring on continuous positive airway pressure adherence. the tele-OSA randomized trial. Am J Respir Crit Care Med 2018;197(1):117-26.

10. FAIRHealth A. Multilayered Analysis of telehealth 2019. Available at: https://s3.amazonaws.com/media2. fairhealth.org/whitepaper/asset/A\%20Multilayered\% 20Analysis\%20of\%20Telehealth\%20-\%20A\%20FAIR $\% 20 \mathrm{Health} \% 20$ White\%20Paper.pdf. Accessed January 15, 2020.

11. Day S, Gambon E. In 2019, digital health celebrated six IPOs as venture investment edged off record highs. Rock Health. Available at: https://rockhealth.com/ reports/in-2019-digital-health-celebrated-six-ipos-asventure-investment-edged-off-record-highs/. Accessed January 15, 2020.

12. Barnett ML, Mehrotra A, Landon BE. Covid-19 and the upcoming financial crisis in health care. NEJM Catal 2020. 